\title{
A pulse phase-dependent spectroscopic study of Vela X-1 in the 8-100 keV band
}

\author{
A. La Barbera ${ }^{1}$, A. Santangelo ${ }^{1}$, M. Orlandini ${ }^{2}$, and A. Segreto ${ }^{1}$ \\ 1 Istituto di Astrofisica spaziale e Fisica Cosmica, C.N.R., sezione di Palermo, via Ugo La Malfa, 153, 90146 Palermo, Italy \\ 2 Istituto di Astrofisica spaziale e Fisica Cosmica, C.N.R., sezione di Bologna, via Piero Gobetti, 101, 40129 Bologna, Italy
}

Received 31 October 2002 / Accepted 23 December 2002

\begin{abstract}
We present a pulse phase resolved study of the 8-100 keV X-ray spectrum of the High Mass X-ray binary Vela X-1, observed by the BeppoSAX satellite. The continuum part of the spectrum is found to be strongly dependent on pulse phase and evidence for saturated and unsaturated Comptonization is found. We confirm the presence of a phase dependent absorption-like feature detected at $\sim 55 \mathrm{keV}$ that we interpret as due to cyclotron resonant scattering. The equivalent width of the feature ranges from $\sim 10$ to $\sim 40 \mathrm{keV}$ whereas its width varies from $\sim 5$ to $\sim 10 \mathrm{keV}$. The energy of the cyclotron line, whose values range from $\sim 50$ to $\sim 60 \mathrm{keV}$, is strongly correlated with the trend of the luminosity intensity of the pulsed profile. With regard to the early reported cyclotron absorption at $\sim 25 \mathrm{keV}$ we do not find strong evidence of it in our BeppoSAX observations.
\end{abstract}

Key words. pulsars: individual: Vela X-1 - stars: neutron - stars: magnetic fields - X-rays: binaries

\section{Introduction}

Vela X-1 (4U 0900-40) is an eclipsing high mass X-ray binary system (HMXB) formed by the massive $\left(M=23 M_{\odot}\right.$ and $R=34 R_{\odot}$ ) B0.5 Ib supergiant HD 77581 (Brucato \& Kristian 1972; Nagase 1989) and a $\sim 1.7 M_{\odot}$ (van Paradijs et al. 1977; Barziv et al. 2001) neutron star (NS). The inferred mass loss rate is $>10^{-7} M_{\odot} \mathrm{yr}^{-1}$ (Sato et al. 1986; Sako et al. 1999) with a terminal wind velocity of $\sim 1700 \mathrm{~km} \mathrm{~s}^{-1}$ (Dupree et al. 1980). The NS is thought to be deeply embedded within the acceleration zone of the stellar wind, in fact the NS orbits the center of mass of the system at a mean distance of $53.4 R_{\odot}$ (only about 0.6 stellar radii from the photosphere of the companion) with a period of 8.96 days in a slightly eccentric orbit $(e \sim 0.09)$.

The NS shows 283 s X-ray pulsations. The pulse period has been found to vary on all timescales from days to years. These variations, described by a random walk in pulse frequency (Deeter et al. 1989), are ascribed to fluctuations in the transfer of angular momentum by the wind accretion. However this scenario presents many problems. In fact current models of spherical accretion are insufficient to explain the observed long term pulse period changes because of the small rate of angular moment transfer (Livio et al. 1986; Soker et al. 1986; Anzer et al. 1987). Although the presence of an accretion disk has been invoked by some authors (see e.g. Börner et al. 1987), up to date experiments have not confirmed any disk.

The spectrum of Vela $\mathrm{X}-1$ is rather complex. White et al. (1983), using HEAO 1 A2 data and more recently

Send offprint requests to: A. La Barbera, e-mail: nino.labarbera@pa.iasf.cnr.it
Kretschmar et al. (1996) based on data from HEXE and TTM on board mir-KVANT modelled the spectrum with a power law with an exponential cutoff beyond $\sim 20 \mathrm{keV}$, whereas Mihara (1995) fitted the 10-38 keV Ginga spectrum using the Negative Positive Exponential (NPEX) model. A considerable photo electric absorption is present at lower energies varying whit orbital phase and sometimes erratically on short timescales (see e.g. Haberl \& White 1990). A strong iron fluorescence emission line at $\sim 6.4 \mathrm{keV}$ and an absorption edge at $\sim 7.2 \mathrm{keV}$ (Nagase et al. 1986) have also been reported.

Kendziorra et al. (1992), using data from HEXE, firstly reported the presence of an absorption-like feature at $\sim 55 \mathrm{keV}$, that the authors interpreted as due to cyclotron resonance scattering. Makishima et al. (1992) reported an absorption feature at $\sim 25 \mathrm{keV}$ from Ginga data, while Choi et al. (1996), also in Ginga data, found the feature at $\sim 32 \mathrm{keV}$. Kretschmar et al. (1997), studying 2-120 keV phase averaged spectra, found indication for absorption features at $\sim 23$ and $\sim 45 \mathrm{keV}$. More recently, observations by RXTE supported the existence of both lines (55 and $25 \mathrm{keV}$ ) (Kreykenbohm et al. 1999, 2002), while the phase averaged spectrum observed with BeppoSAX showed only one absorption line at $\sim 55 \mathrm{keV}$ (Orlandini et al. 1998).

Due to resonant scattering of electrons in the Landau levels, cyclotron features provide a powerful tool for directly measuring the high ( $B \geq 10^{12}$ Gauss) magnetic field strengths of accreting neutron stars in $\mathrm{X}$-ray binaries. Because the electron cyclotron energy $E_{\text {cycl }} \simeq 11.6 B_{12} \mathrm{keV}$, where $B_{12}$ is the magnetic field strength in unit of $10^{12}$ Gauss, these features are expected to be observed at hard X-ray energies. Emission from 
accreting HMXB is thought to be strongly influenced by magnetic fields. The knowledge of the magnetic field strength and its dependence on the pulse phase is, therefore, key in order to understand the physics and geometry of the emission from HMXB (Nagel 1981; Meszaros \& Nagel 1985a, 1985b).

The main aim of this paper is the study of the pulse phase dependence of the high energy $(8-100 \mathrm{keV})$ spectrum of the source. The phase dependence of the cyclotron absorption feature is also studied. Our findings permits a deeper description of the geometric structure of the magnetic field around the star and consequently a deeper understanding of the accretion column physics at the hot spots. This study is based on several unpublished BeppoSAX observations.

\section{BeppoSAX observations and data reduction}

The BeppoSAX satellite (Boella et al. 1997a) has been active in the X-ray sky from April 1996 till April 2002. The payload was composed by four Narrow Field Instrument (NFIs) and two Wide Field Cameras (Jager et al. 1997). Besides the Low-Energy Concentrator Spectrometer (LECS, 0.1-10 keV, Parmar et al. 1997) and the Medium-Energy Concentrators Spectrometer (MECS, 2-10 keV, Boella et al. 1997b), the NFIs comprise the two collimated high energy detectors, the High Pressure Gas Scintillation Proportional Counter (HPGSPC, 4$60 \mathrm{keV}$, FWHM energy resolution of $8 \%$ at $10 \mathrm{keV}$ and $5.5 \%$ at $20 \mathrm{keV}$, Manzo et al. 1997), and the Phoswich Detection System (PDS, 15-200 keV, FWHM energy resolution of $24 \%$ at $20 \mathrm{keV}$, and $14 \%$ at $60 \mathrm{keV}$, Frontera et al. 1997). The two collimated instruments (HPGSPC and PDS) were operated in rocking mode with a $96 \mathrm{~s}$ stay time in order to monitor the background along the orbit. Details on data reduction and spectral analysis can be found in the "handbook for BeppoSAX NFIs spectral analysis". In the handbook values for normalization constants for the BeppoSAX NFIs are reported.

The BeppoSAX satellite observed Vela X-1 with NFIs several times from July 14th 1996 to December 25th 1997. In Table 1 we report the complete list of the BeppoSAX observations of Vela X-1 and the relative orbital phases. In this study we used the observations with OP numbers 1428, 1430, 1431 and 3193 (see Table 1), for a total duration of $\sim 265 \mathrm{ks}$, covering about the $0.35-0.65$ orbital phase with phase zero corresponding to mid-eclipse.

Those observations were selected on the basis of a rather uniform luminosity $\left(L_{8-100} \simeq 2.7 \times 10^{36} \mathrm{erg} \mathrm{s}^{-1}\right.$, assuming a distance of $1.9 \mathrm{kpc}$ (Sadakane et al. 1985)) and a low spectral variability. Strong spectral variability could seriously affect the resulting spectra making difficult if not meaningless the determination of the spectrum continua, which is critical for line studies.

\section{Time analysis}

To obtain the energy-resolved pulse profiles and phaseresolved energy spectra, the photon arrival times were first corrected to the Solar System barycenter. By using period folding (Leahy et al. 1983), we searched for the pulse period for each observation individually. Considering the relative long orbital
Table 1. Log of the BeppoSAX observations of Vela X-1.

\begin{tabular}{lllll}
\hline \hline Obs. & NFI OP & Start time & $\begin{array}{l}\text { Obs. time } \\
(\mathrm{ksec})\end{array}$ & \\
\hline 1 & 660 & Jul. 14 1996 06:53:39 & 48 & $0.28-0.35$ \\
2 & 1428 & Dec. 23 1996 05:59:43 & 18 & $0.35-0.38$ \\
2 & 1430 & Dec. 23 1996 11:14:18 & 96 & $0.38-0.50$ \\
2 & 1431 & Dec. 24 1996 15:00:58 & 99 & $0.51-0.63$ \\
2 & 1432 & Dec. 25 1996 18:47:38 & 15 & $0.63-0.65$ \\
3 & 1438 & Dec. 27 1996 10:14:58 & 7 & $0.82-0.83$ \\
4 & 2983 & Nov. 24 1997 13:05:10 & 100 & $0.83-0.99$ \\
4 & 2984 & Nov. 25 1997 16:51:50 & eclipse \\
5 & 3192 & Dec. 24 1997 14:31:37 & 100 & $0.22-0.35$ \\
5 & 3193 & Dec. 25 1997 18:18:17 & 52 & $0.35-0.42$ \\
\hline \multicolumn{5}{l}{ Ephemerides from Nagase (1989). }
\end{tabular}

period, the long pulse period and the shortness of the single set of data with respect to the orbital period, we ignored any correction due to the NS orbital motion. As an example the corresponding pulse profiles of the OP1430 data as a function of the energy, obtained by folding the NFIs light curves, are shown in Figs. 1-4.

The source is found to pulsate up to $\sim 100 \mathrm{keV}$. Below $\sim 7.0 \mathrm{keV}$ the pulsed light curve exhibits a five peaks structure organized in two broad pulses containing three peaks (phase 0.0-0.55) and two peaks (phase 0.55-1.0) respectively. The relative size of the peaks is not constant in the different energy bands, changing the relative importance (Orlandini 1993). In addition to the major peaks, other energy dependent minor features are observed: for example the one at phase $\sim 0.0$ in the 2.8-7.0 keV energy range.

For energies greater than $7.0 \mathrm{keV}$ the five peaks structure of the pulsed light curve evolves in two broad single peaks. We define the peak at phase $0.05-0.55$ as first peak, while we define the one at phase $0.55-1.05$ as second peak. Other minor features are still observable, superimposed to the main double peaked feature. The high energy double peak structure appears to be stable enough with respect to the time.

To test spectral variability in the high energy range, we studied the time evolution of the hardness ratio for each selected observation and for the HPGSPC and PDS instruments (20-30 keV/7-20 keV for the HPGSPC and 30-100 keV/15$30 \mathrm{keV}$ for the PDS). Hardness ratios are rather constant indicating that the spectra which we analyzed were free from time variability effects.

\section{Spectral analysis}

Cyclotron line(s) in Vela X-1 (Orlandini et al. 1998; Kreykenbohm 2002 and references therein) is expected above $20 \mathrm{keV}$. We therefore selected the phase intervals based on the $15-100 \mathrm{keV}$ pulse profile as observed by the PDS. These intervals, labelled with capital alphabetic characters (see also Table 2), are shown in Fig. 5. Phase A and Phase F correspond to the two minima of pulsed light curve, with the intensity in Phase F lower than in Phase A; Phase B and Phase C, Phase G and Phase $\mathrm{H}$ correspond to the rising edges of the first and second peak respectively; Phase D and Phase I correspond to 

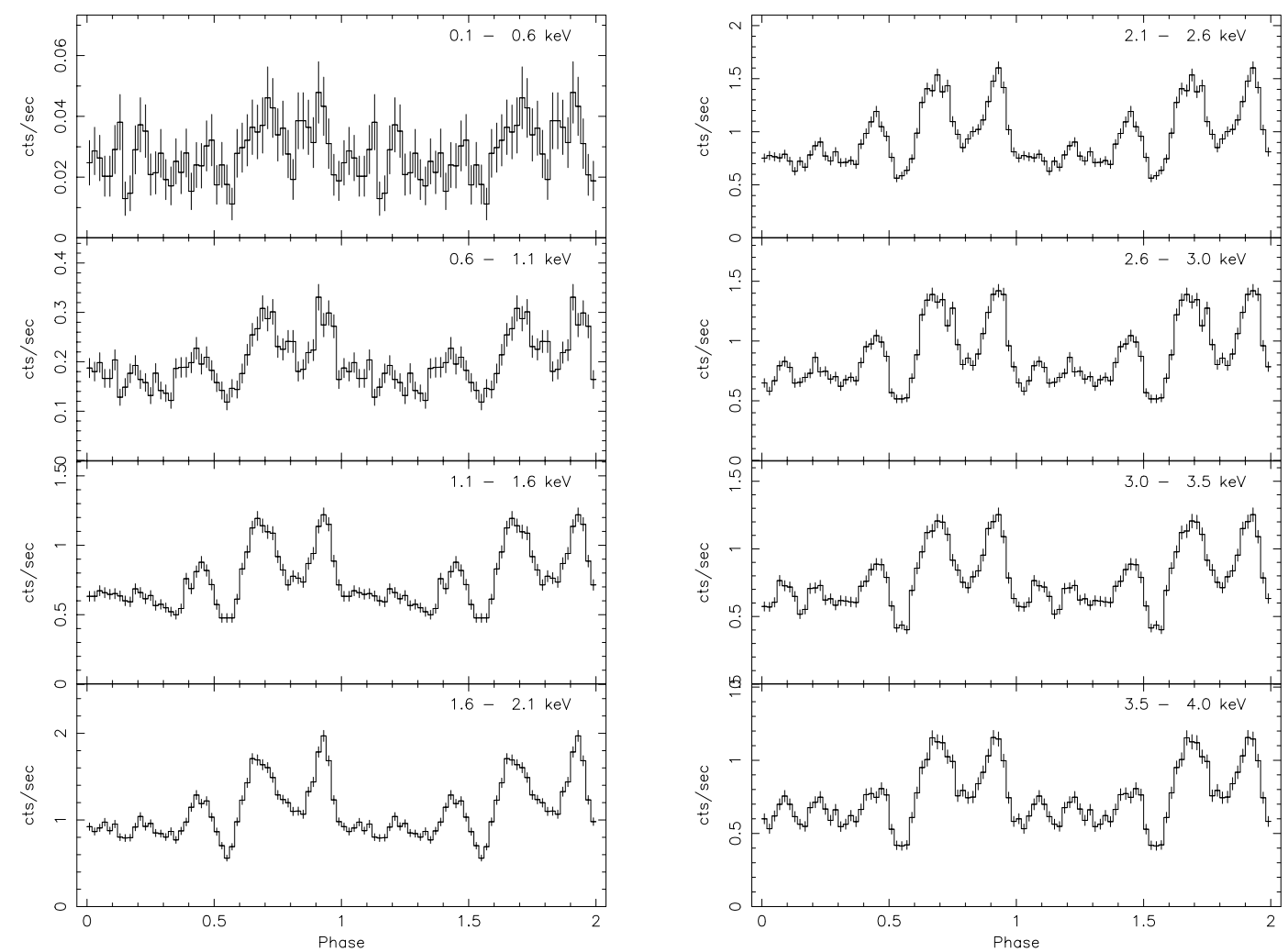

Fig. 1. Background subtracted pulse profiles of the LECS data as a function of energy relative to obs. OP 1430.
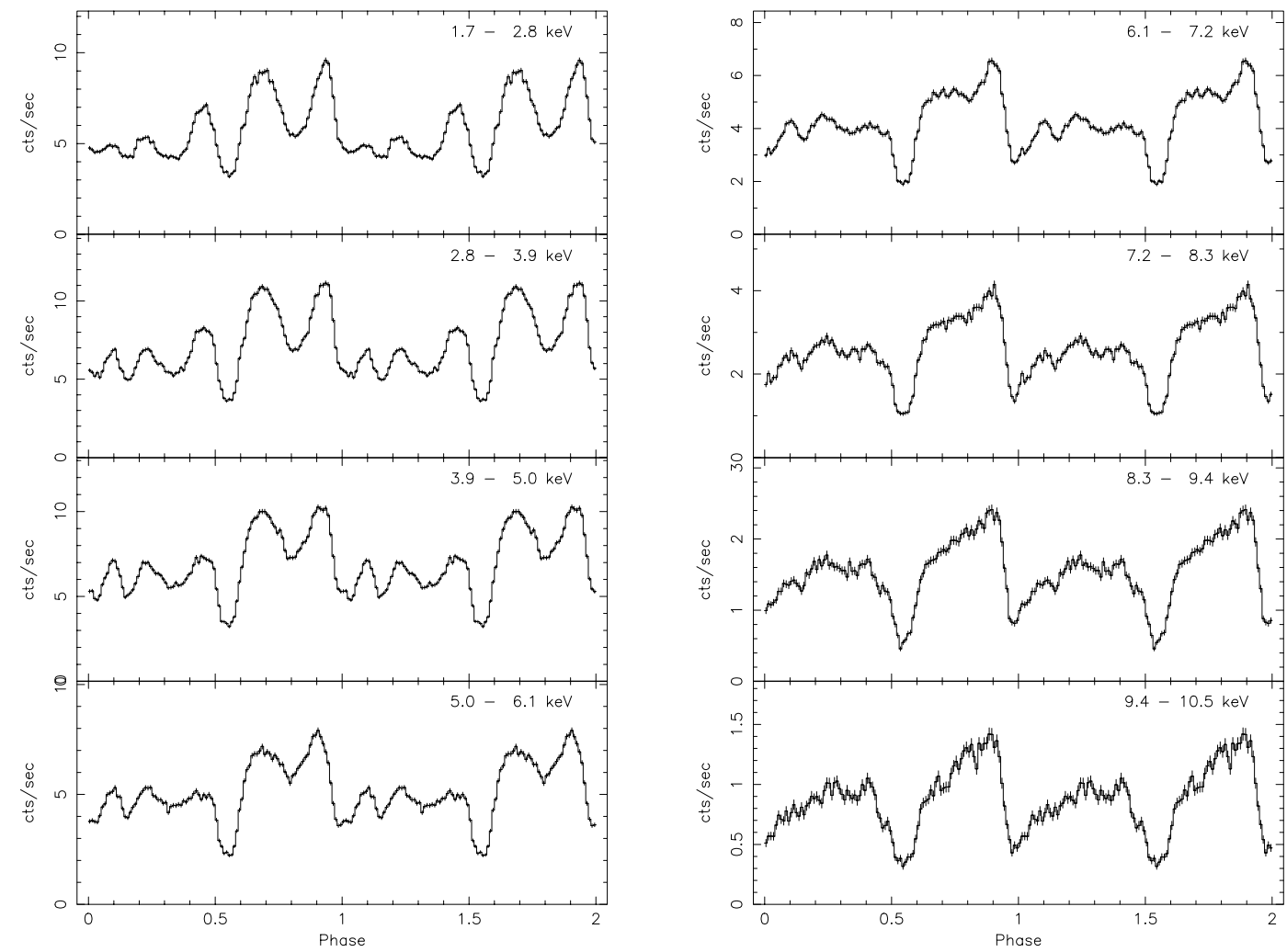

Fig. 2. Background subtracted pulse profiles of the MECS data as a function of energy relative to obs. OP 1430.

the two maxima; eventually Phase E and Phase J correspond to the two descents. To be sure that phase resolved spectra did not vary with time, for each phase bin, we studied the ratios between two corresponding phase selected spectra relative to two different observations. These ratios are quite constant with respect to energy. For each phase bin, summing 

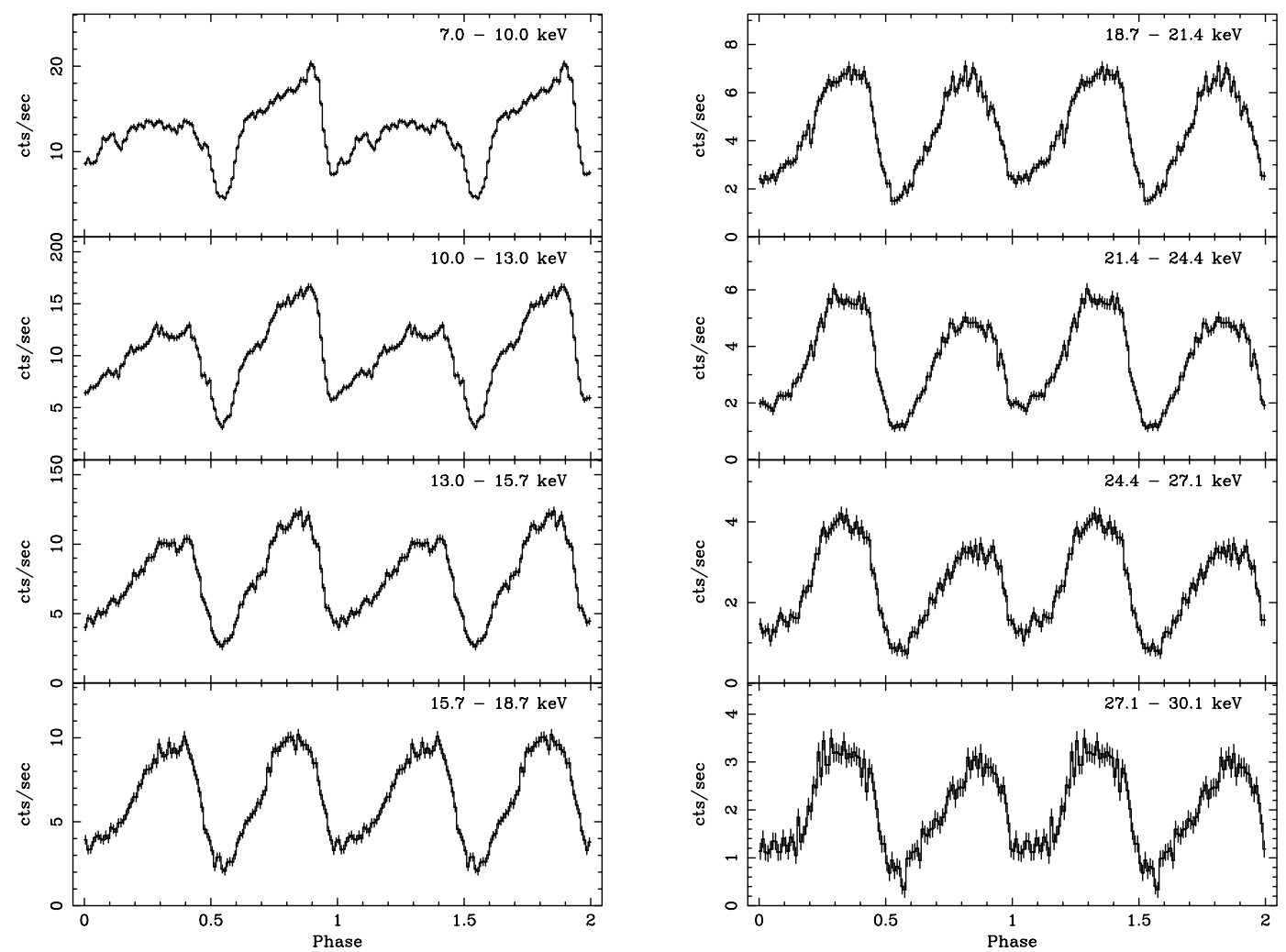

Fig. 3. Background subtracted pulse profiles of the HPGSPC data as a function of energy relative to obs. OP 1430.
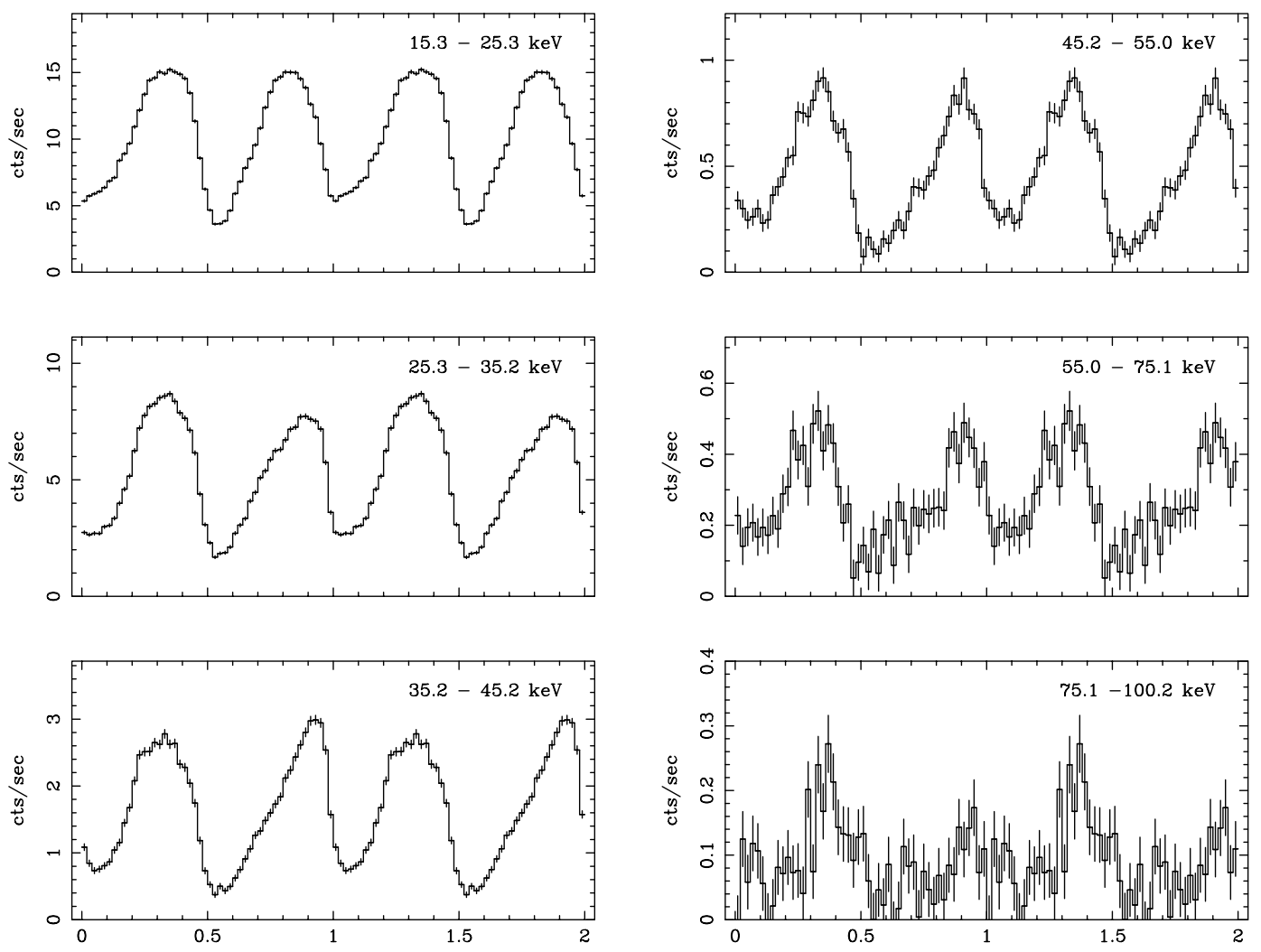

Fig. 4. Background subtracted pulse profiles of the PDS data as a function of energy relative to obs. OP 1430.

all the selected observations, background subtracted phase resolved $(8-100 \mathrm{keV})$ spectra were obtained for both the HPGSPC and PDS.
We tried many different continuum models to find a unique model for all the phases. However, it was not possible to fit the 


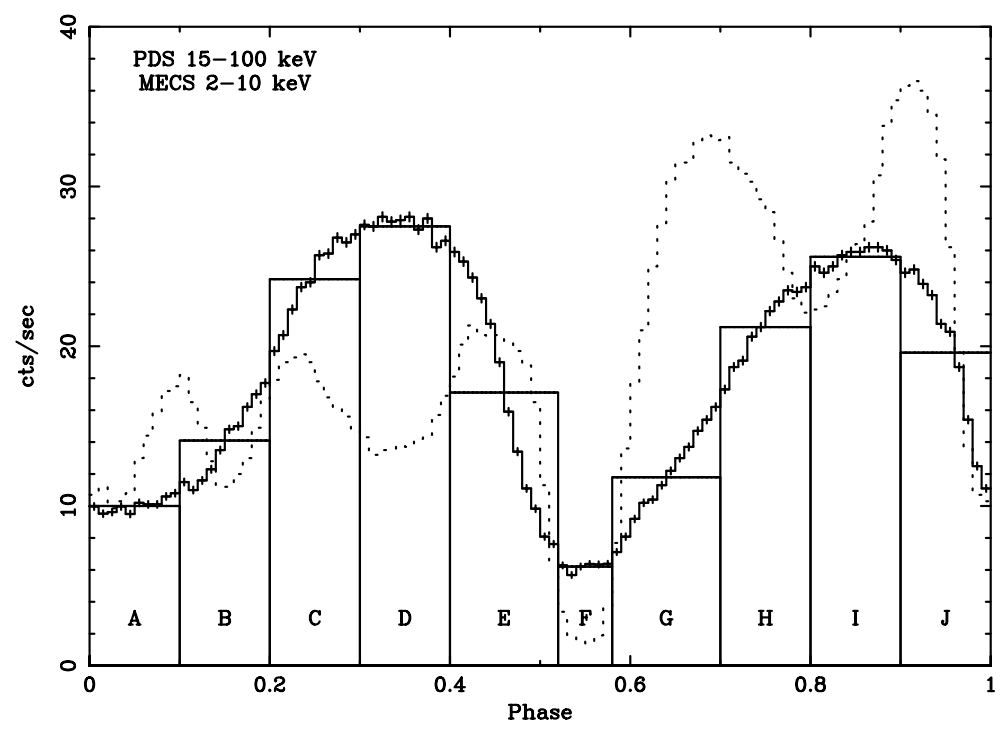

Fig. 5. Phase selected intervals superimposed to the PDS pulse profile. For comparison the MECS pulse profile in arbitrary units is also plotted (dotted line).

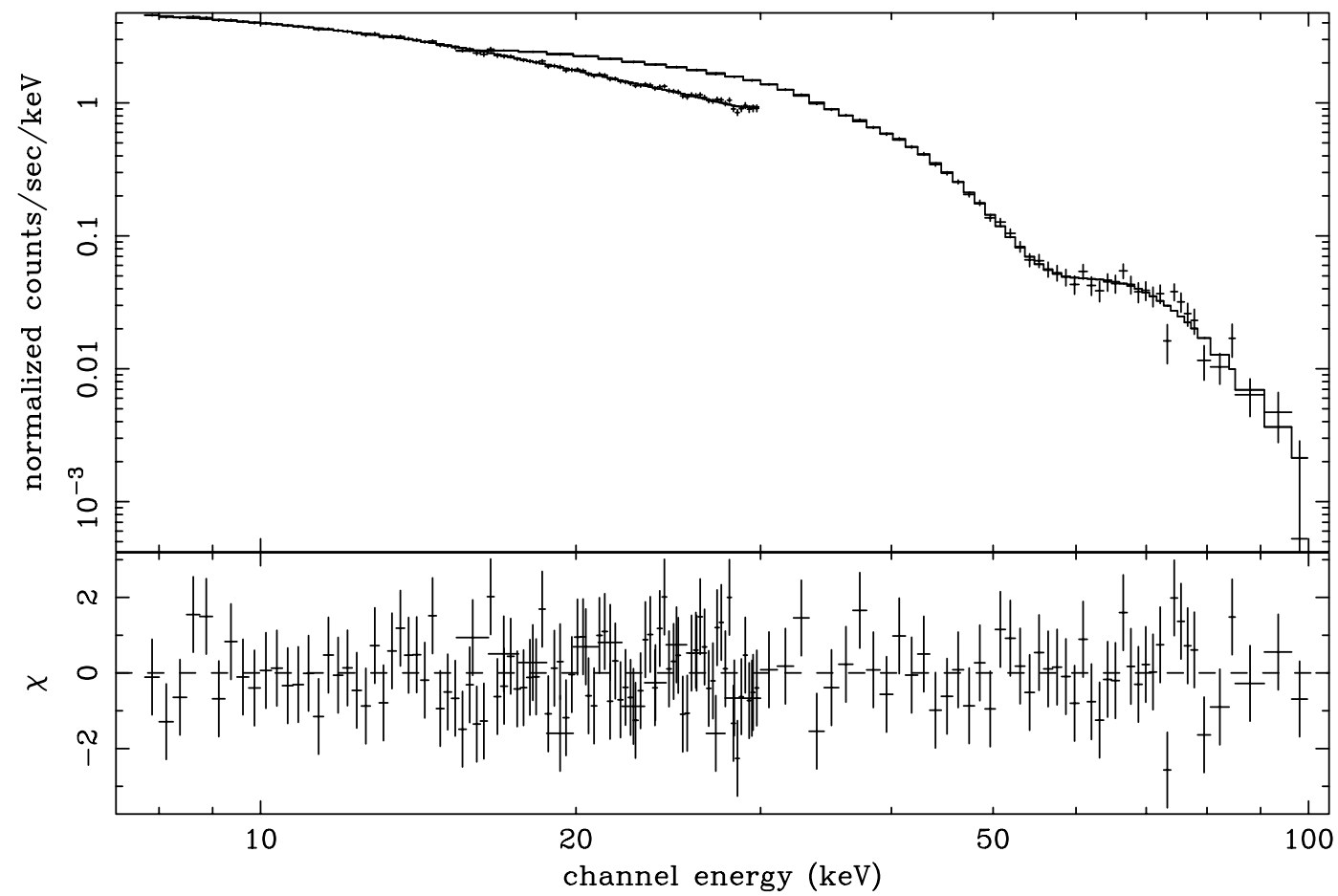

Fig. 6. BeppoSAX Vela $\mathrm{X}-1$ spectrum relative to pulse Phase J. Top panel: source spectrum (crosses) and best fit model (histogram) (see Table 5), bottom panel: residuals from the best fit model.

phase resolved spectra relative to the different phase intervals by using a unique continuum model. In the following a detailed description of the spectra of each pulse phase is reported. As an example of the goodness of our data statistics we plot in Fig. 6 the spectrum of Phase $\mathbf{J}$ (top panel) with the residuals (bottom panel) relative to the best model used to fit the data. In Fig. 7 we plot the energy unfolded spectra relative to each selected phase with the best model superimposed while in Tables 3-5 we report the values of the best fit parameters and the resulting $\operatorname{minima} \chi_{\text {d.o.f. }}^{2}$.
The spectral continuum of the Phases A and B: Spectra of Phase A, that corresponds to the first minimum of the pulsed light curve, and Phase B, the first part of the ascent edge of the first peak, were well fitted simply by a power law modified by a high energy cutoff (CUTOFFPL) of the form:

$N(E)=N O R M E^{-\alpha} \exp \left(-E / E_{\text {cut }}\right)$

where $\alpha$ is the photon index and $E_{\text {cut }}$ is the energy cutoff.

The spectral continuum of the Phases C: This simple model failed in fitting the spectral continuum of Phase $\mathrm{C}$, the second 
Table 2. Pulse phase selection.

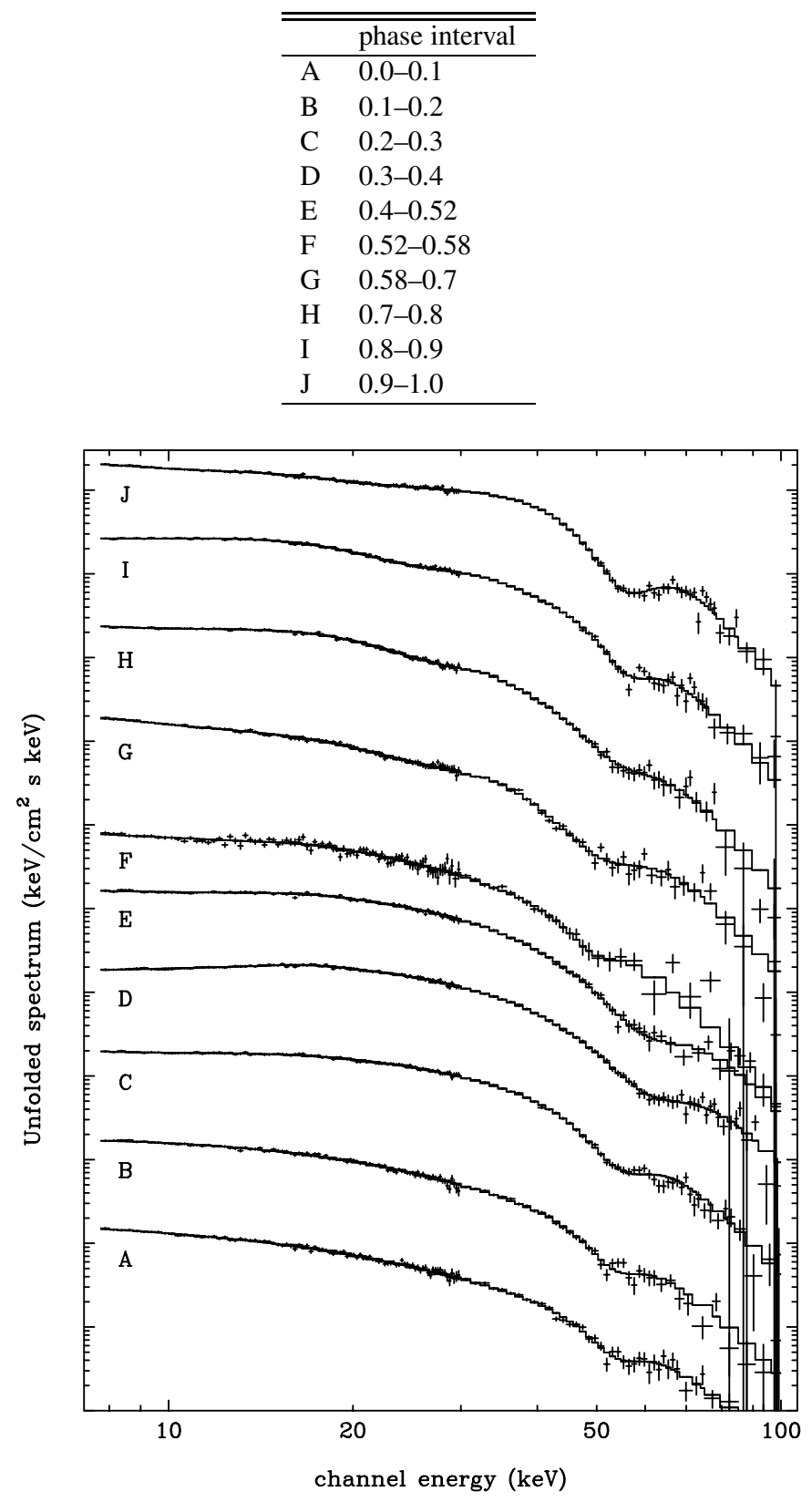

Fig. 7. Unfolded spectra of Vela X-1 relative to all pulse phases. Each spectrum has been shifted for clarity.

part of the first peak rising edge. In order to obtain a reasonable fit a second softer component was necessary. We therefore used the CUTOFFPL model plus a second softer power law.

The spectral continuum of the Phases D, E, F: These phases correspond to the first peak of the pulsed light curve till the second minimum at Phase F. In these phases the spectral continuum has a more complex form that cannot be fitted with a CUTOFFPL model. To have a more physical description of the continuum spectrum we used a model (that we define POWEXP) that evolves from NPEX model (Mihara 1995; Makishima 1999) and is a simple analytical approximation for

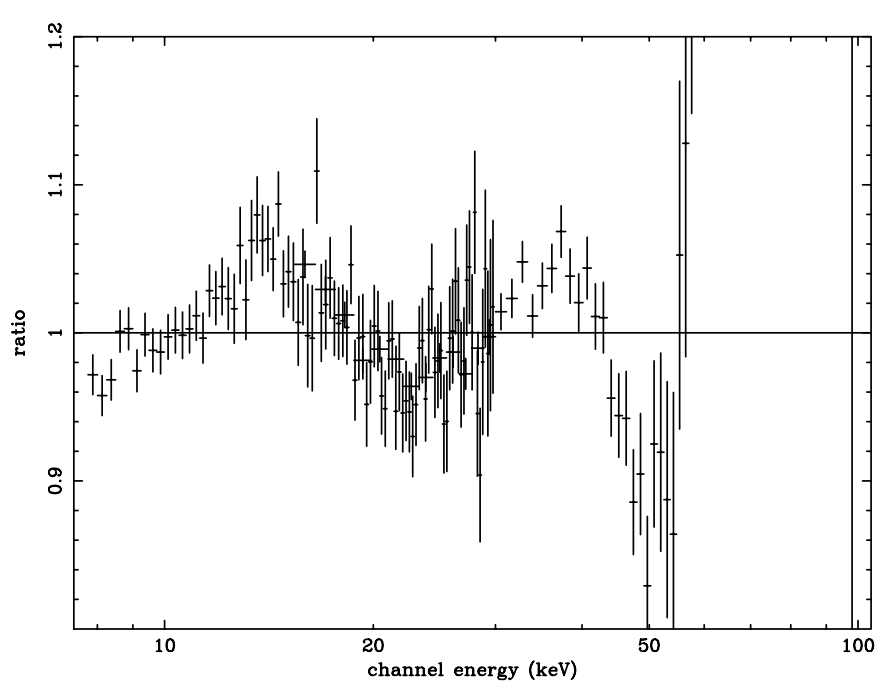

Fig. 8. Ratio between the spectral continuum model and data relative to Phase J. At $\sim 50 \mathrm{keV}$ an absorption feature is evident. A complex residual is present below $30 \mathrm{keV}$, which could be interpreted or as an excess between 10 and $20 \mathrm{keV}$ or as an absorption between 20 and $30 \mathrm{keV}$.

the spectrum produced by the upscattering of soft photons in a hot plasma of temperature $T$ (Sunyaev \& Titarchuck 1980):

$f(E) \propto E^{-\alpha}$ if $E \leq E_{\text {cut }}-W / 2$
$f(E) \propto E^{2} \exp \left(-E / k T_{\mathrm{e}}\right)$ if $E \geq E_{\text {cut }}+W / 2$

(smoothed between $E_{\text {cut }}-W / 2$ and $E_{\text {cut }}+W / 2$ with a polynomial). At low energies, the model reduces to the power law. As the energy increases, at $E \sim k T_{\mathrm{e}}$ the positive slope with $\alpha=2$ models the Wien tail. At $E>k T_{\mathrm{e}}$, the model exhibits a thermal exponential decay. We obtained a quite uniform behavior of the spectral parameters in all the phases. The photon index is $\sim 1, E_{\text {cut }}$ is $\sim 16 \mathrm{keV}$ and $k T_{\text {e }}$, i.e. the folding energy $\left(E_{\text {fold }}\right)$, is $\sim 10 \mathrm{keV}$.

The spectral continuum of the Phases G, H, I, J: These phases correspond to the second peak of the pulsed light curve being Phase I the maximum. We still modeled the continuum with the POWEXP model. In order to search for possible additional components, in Fig. 8 we plot the ratio between the model of spectral continuum and the data relative to Phase J. Clear residuals are present. At $\sim 50 \mathrm{keV}$ an absorption feature is evident. We will discuss this feature in the following when we describe the high energy cyclotron absorption feature. Moreover a complex residual is present below $30 \mathrm{keV}$, which could be interpreted or as an excess between 10 and $20 \mathrm{keV}$ or an absorption between 20 and $30 \mathrm{keV}$. We tried to correct the continuum adopting the absorption interpretation, but we were not able to obtain good fits (see the paragraph in which we discuss the possible presence of a low energy cyclotron line). Then we modelled the residuals at low energies (below $20 \mathrm{keV}$ ) with a large Gaussian in emission centered at $\sim 13 \mathrm{keV}$. In Fig. 9 we plot the unfolded spectrum relative to Phase $\mathbf{J}$ in which it is possible to evaluate the contribution due to the Gaussian bump correction. Also in these phases we obtained a quite uniform 
Table 3. Best fits results for the Vela X-1 spectra of the pulse Phases A, B and C. The model used is: CYCLOTRON (shaped as a Gaussian in absorption) $\times$ [CUTOFFPL + POWER LAW (only for Phase C)]. A constant factor has been taken into account in order to accord HPGSPC and PDS data. The constant was fixed to 1 for the HPGSPC while it was left free for PDS. The uncertainties are given at $3 \sigma$ of confidence.

\begin{tabular}{|c|c|c|c|}
\hline parameter & Phase A & Phase B & Phase C \\
\hline CYCL. Equiv. Width (keV) & $13 \pm 4$ & $13 \pm 4$ & $20 \pm 3$ \\
\hline CYCL. Energy Line (keV) & $53.5 \pm 2$ & $53 \pm 2$ & $54.4 \pm 0.7$ \\
\hline CYCL. $\sigma(\mathrm{keV})$ & $5.5 \pm 1.5$ & $5.5 \pm 1.5$ & $7.0 \pm 0.8$ \\
\hline CUTOFFPL Phot. index & $0.85 \pm 0.05$ & $0.52 \pm 0.05$ & $-0.37 \pm 0.08$ \\
\hline CUTOFFPL Folding en. (keV) & $14.1 \pm 0.6$ & $11.8 \pm 0.4$ & $9.7 \pm 0.3$ \\
\hline CUTOFFPL Norm. $^{a}$ & $0.19 \pm 0.02$ & $0.12 \pm 0.01$ & $(1.95 \pm 0.3) \times 10^{-2}$ \\
\hline POW. LAW Phot. index & & & $4.0 \pm 1.5$ \\
\hline POW. LAW Norm. ${ }^{a}$ & & & $23 \pm 3$ \\
\hline PDS Constant & $0.80 \pm 0.01$ & $0.82 \pm 0.01$ & $0.843 \pm 0.005$ \\
\hline reduced $\chi^{2}$ & 1.019 & 1.076 & 1.005 \\
\hline d.o.f. & 158 & 158 & 156 \\
\hline
\end{tabular}

${ }^{a}$ Photons $/ \mathrm{keV} / \mathrm{cm}^{2} / \mathrm{s}$ at $1 \mathrm{keV}$.

Table 4. Best fits results for the Vela X-1 spectra of the pulse Phases D, E and F. The model used is: CYCLOTRON (shaped as a Gaussian in absorption) $\times$ [POWEXP]. A constant factor has been taken into account in order to accord HPGSPC and PDS data. The constant was fixed to 1 for the HPGSPC while it was left free for PDS. The uncertainties are given at $3 \sigma$ of confidence.

\begin{tabular}{|c|c|c|c|}
\hline parameter & Phase D & Phase E & Phase F \\
\hline CYCL. Equiv. Width (keV) & $37 \pm 5$ & $38 \pm 7$ & $9 \pm 4$ \\
\hline CYCL. Energy Line (keV) & $59.5 \pm 1$ & $58.5 \pm 1.5$ & $49_{-3}^{+5}$ \\
\hline CYCL. $\sigma(\mathrm{keV})$ & $12 \pm 1$ & $11.5 \pm 1$ & $5 \pm 3$ \\
\hline POWEXP Phot. index & $0.815 \pm 0.015$ & $1.11 \pm 0.03$ & $1.35 \pm 0.05$ \\
\hline POWEXP Cutoff en. (keV) & $16.6 \pm 0.3$ & $16.2 \pm 0.7$ & $16 \pm 1$ \\
\hline POWEXP Folding en. (keV) & $11.4 \pm 0.2$ & $10.5 \pm 0.2$ & $9.1 \pm 0.2$ \\
\hline POWEXP Width (keV) & $2 \pm 1.5$ & $3 \pm 1.5$ & $2 \pm 2$ \\
\hline POWEXP Norm. ${ }^{a}$ & $(1.27 \pm 0.03) \times 10^{-2}$ & $(9.2 \pm 0.6) \times 10^{-3}$ & $(3.7 \pm 0.5) \times 10^{-3}$ \\
\hline PDS Constant & $0.822 \pm 0.006$ & $0.816 \pm 0.7$ & $0.83 \pm 2$ \\
\hline reduced $\chi^{2}$ & 1.069 & 1.023 & 1.209 \\
\hline d.o.f. & 155 & 155 & 155 \\
\hline
\end{tabular}

${ }^{a}$ Photons $/ \mathrm{keV} / \mathrm{cm}^{2} / \mathrm{s}$ at $1 \mathrm{keV}$.

Table 5. Best fits results for the Vela X-1 spectra of the pulse Phases G, H, I and J. The model used is: CYCLOTRON (shaped as a Gaussian in absorption) $\times$ [POWEXP + GAUSSIAN]. A constant factor has been taken into account in order to accord HPGSPC and PDS data. The constant was fixed to 1 for the HPGSPC while it was left free for PDS. The uncertainties are given at $3 \sigma$ of confidence.

\begin{tabular}{|c|c|c|c|c|}
\hline parameter & Phase G & Phase $\mathrm{H}$ & Phase I & Phase J \\
\hline CYCL. Equiv. Width (keV) & $31 \pm 6$ & $21_{-7}^{+9}$ & $16 \pm 5$ & $36 \pm 12$ \\
\hline CYCL. Energy Line (keV) & $50 \pm 2$ & $53.2 \pm 1.5$ & $55 \pm 1$ & $55.2 \pm 0.5$ \\
\hline CYCL. $\sigma(\mathrm{keV})$ & $9 \pm 2$ & $8 \pm 2$ & $6.0 \pm 1.5$ & $9 \pm 2$ \\
\hline POWEXP Phot. index & $2.05 \pm 0.03$ & $1.77 \pm 0.03$ & $1.57 \pm 0.04$ & $1.65 \pm 0.10$ \\
\hline POWEXP Cutoff en. (keV) & $35 \pm 2$ & $33 \pm 1$ & $31.6 \pm 0.5$ & $44 \pm 9$ \\
\hline POWEXP Folding en. (keV) & $9.8_{-0.2}^{+0.6}$ & $9.2 \pm 0.5$ & $9.7 \pm 0.4$ & $9 \pm 2$ \\
\hline POWEXP Width (keV) & $3.5_{-3.5}^{+3}$ & $2_{-2}^{+3}$ & $5 \pm 2$ & $16_{-10}^{+2}$ \\
\hline POWEXP Norm. ${ }^{a}$ & $\left(1.0_{-0.2}^{+0.5}\right) \times 10^{-3}$ & $(2.0 \pm 0.2) \times 10^{-3}$ & $(3.0 \pm 0.15) \times 10^{-3}$ & $\left(1.5_{-0.6}^{+0.9}\right) \times 10^{-3}$ \\
\hline GAUSS. Energy Line (keV) & $12.5 \pm 1.0$ & $12.8 \pm 0.5$ & $11.3 \pm 0.8$ & $13.5 \pm 1.0$ \\
\hline GAUSS. $\sigma(\mathrm{keV})$ & $5.4 \pm 0.7$ & $6.0 \pm 0.3$ & $5.9 \pm 0.5$ & $3.4_{-0.6}^{+0.8}$ \\
\hline GAUSS. norm. ${ }^{b}$ & $\left(2.9_{-0.7}^{+0.1}\right) \times 10^{-2}$ & $(9 \pm 1) \times 10^{-2}$ & $(12 \pm 2) \times 10^{-2}$ & $\left(1.0_{-0.2}^{+0.5}\right) \times 10^{-2}$ \\
\hline PDS Constant & $0.845 \pm 0.010$ & $0.834 \pm 0.006$ & $0.835 \pm 0.005$ & $0.835 \pm 0.010$ \\
\hline reduced $\chi^{2}$ & 1.117 & 1.011 & 1.074 & 0.858 \\
\hline d.o.f. & 153 & 153 & 153 & 153 \\
\hline
\end{tabular}

${ }^{a}$ Photons $/ \mathrm{keV} / \mathrm{cm}^{2} / \mathrm{s}$ at $1 \mathrm{keV}$.

${ }^{b}$ Total photons $/ \mathrm{cm}^{2} / \mathrm{s}$. 


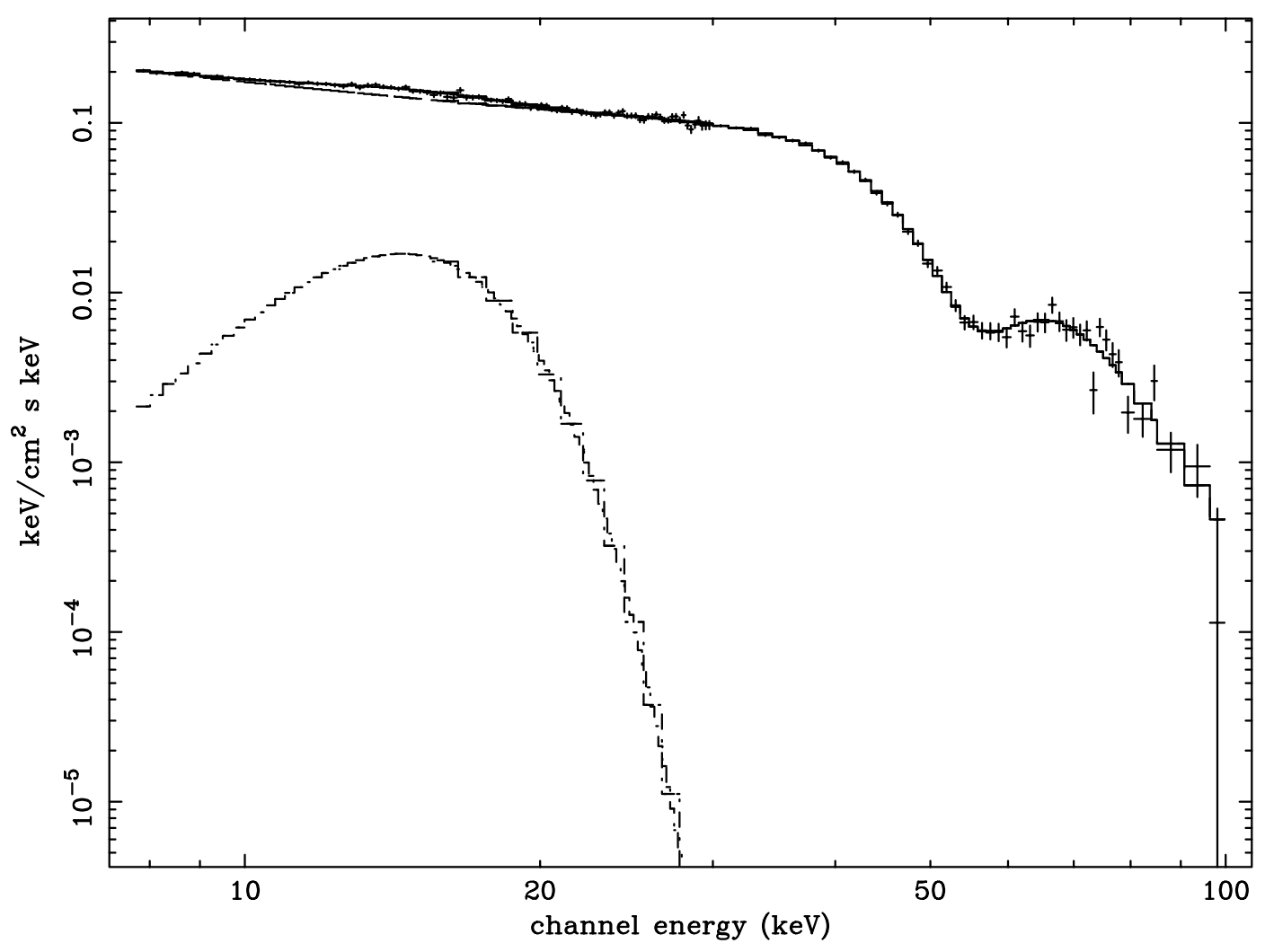

Fig. 9. The unfolded spectrum relative to Phase J. Note the Gaussian bump correction at energies below $20 \mathrm{keV}$.

behavior of the spectral parameters. The photon indexes range from $\sim 1.5$ to $\sim 2, E_{\text {cut }}$ ranges roughly between 30 and $40 \mathrm{keV}$ and $E_{\text {fold }}$ is $\sim 10 \mathrm{keV}$ as previously obtained for the Phases C, $\mathrm{D}, \mathrm{E}$ and $\mathrm{F}$.

The high energy cyclotron line: An additional feature centered around $\sim 55 \mathrm{keV}$ was clearly apparent in the residuals of the PDS spectra at all phases. This prompted us to introduce an absorption feature, in the model as a Gaussian filter in absorption

$G(E)=1-D \times \exp \left(-\left(E-E_{\text {cycl }}\right)^{2} /\left(2 \sigma^{2}\right)\right)$

where $E_{\text {cycl }}, \sigma$, and $D$ are the centroid energy, width and depth of the feature. The resulting minimum $\chi_{\text {d.o.f. }}^{2}$ for each phase together with the best-fit parameters are reported in Tables 3-5. In order to evidence the role of the cyclotron absorption, in Fig. 10 we plot the spectrum relative to the Phase $\mathrm{J}$ superimposed to the best fit subtracted of the cyclotron absorption (top panel). The ratio data/model is also plotted (bottom panel). The centroid energy of the cyclotron feature as a function of the phase follows the trend of the pulsed curve with a small shift, ranging from 50 to $60 \mathrm{keV}$. With regard to $\sigma$ and the equivalent width $(\mathrm{EW})$ we observe that they have the same behavior with the phase but no clear correlation with the pulsed curve is evident. In particular these parameters have three minima and three maxima. Two minima correspond to the minima of the pulsed curve but the third minimum corresponds to the maximum of the second peaks; moreover two maxima lie at the descents of the luminosity peaks while the third maximum is in correspondence of the ascent of the second peak.
A shallow cyclotron resonance scattering feature at $\sim 25 \mathrm{keV}$ has been reported in literature from many authors (Mihara 1995; Kreykenbohm et al. 2002), who interpreted the line as the fundamental cyclotron line. By using the model continua described in the previous paragraphs no residuals at $\sim 25 \mathrm{keV}$ are observed at any phase. However, in order to check the possible presence in our BeppoSAX observation of this lower energy cyclotron line we studied Phases G, H, I, J to clear if the Gaussian bumps could be an effect of a $25 \mathrm{keV}$ cyclotron absorption. Also in this case we tried to fit the spectral continuum corresponding to these phases with the POWEXP model, but we were not able to obtain good fits. So we fitted the spectra of Phases G and J with the NPEX continuum, also used by the authors above reported (differently of them we permit to spectral indexes to be freely positive or negative) plus two lines, the high energy line at $\sim 55 \mathrm{keV}$ and the low energy line at $\sim 25 \mathrm{keV}$. A power law modified by a high energy cutoff model was used for fitting the spectral continuum of the Phases $\mathrm{H}$ and I. To avoid cusps we did not use the XSPEC multiplicative model HIGHECUT. We used a slightly modified multiplicative model (NHIGH) which is 1 for $E \leq E_{\text {cut }}-W / 2$ while is $\exp \left[-\left[\left(E-E_{\text {cut }}\right) / E_{\text {fold }}\right]\right]$ for $E \geq E_{\text {cut }}+W / 2$. The energy interval of width $W$ between $E_{\text {cut }}-W / 2$ and $E_{\text {cut }}+W / 2$ is connected to the external intervals by a cubic polynomial in order to have a continuous and derivable mathematical function in the whole energy range. Although from a statistical point of view the modelling with two cyclotron lines is equivalent to the one-line model (the probability of chance improvement of the $\chi^{2}$ in the two models, computed from an F-test, is $40 \%$ ), the former is unacceptable because the values of the 


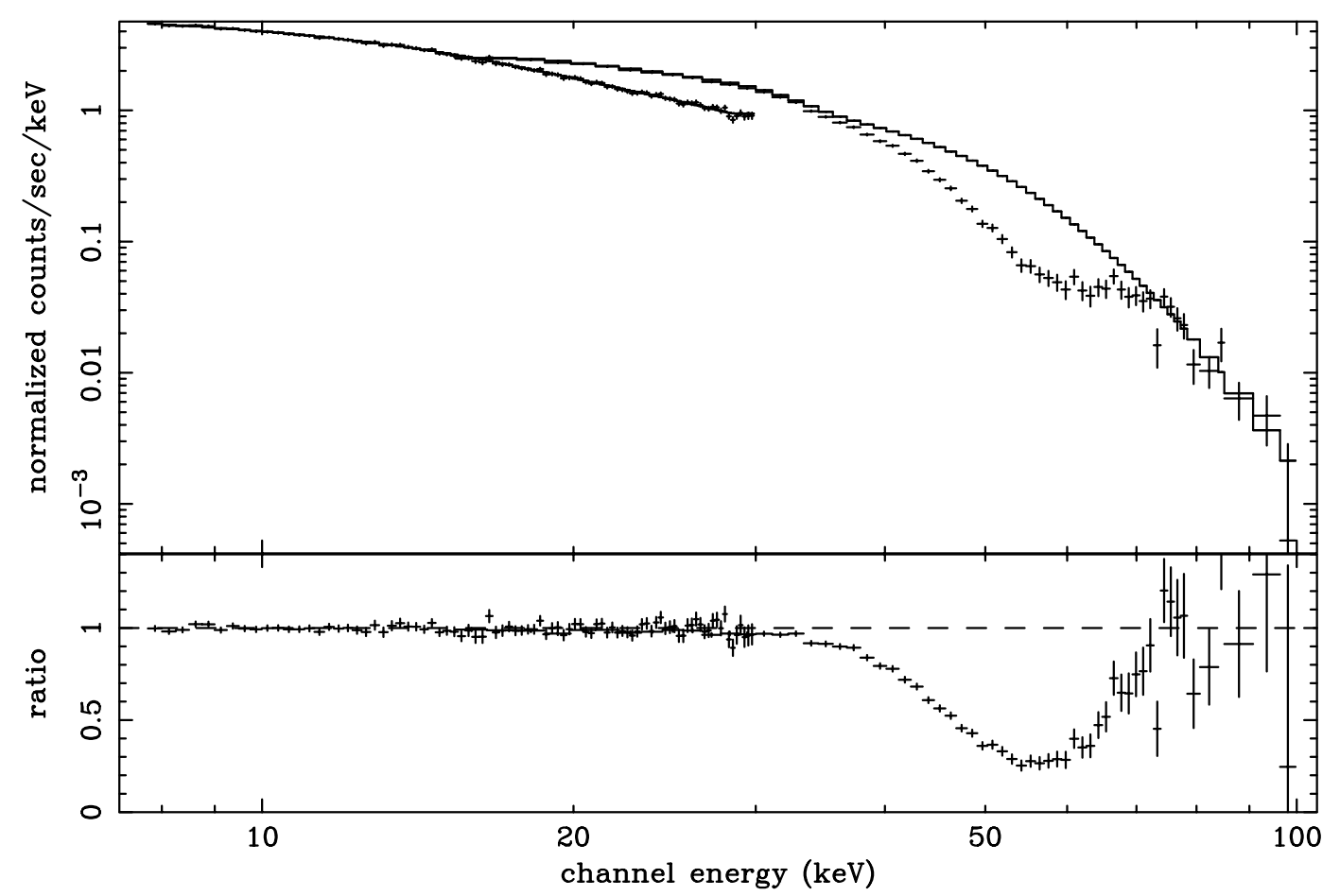

Fig. 10. Top panel: The spectrum relative to Phase $\mathbf{J}$ (crosses) with the best fit subtracted of the cyclotron absorption (histogram). Bottom panel: Ratio between the spectral data and the best fit subtracted of the cyclotron absorption.

normalization constants between the two instrument are in complete disagreement with the ones resulting from extensive calibration and reported in the BeppoSAX handbook. The results of these fits are summarized in Table 6 whereas in Fig. 11 we compare the residuals obtained by using the model containing the Gaussian bump with the residuals obtained by using the model containing the low energy cyclotron absorption.

\section{Discussion}

The 8-100 keV pulse phase dependent spectrum of Vela $\mathrm{X}-1$ has been studied in detail dividing the pulse profile in 10 phase intervals. Phase resolved spectra of Vela X-1 shows a complex structure in all phases. Both continuum and features depend on the pulse phase. In this section first we will discuss the continuum then we will comment on the behavior of the cyclotron resonant feature.

At Phases A and B the $8-100 \mathrm{keV}$ continuum is simply described by a single XSPEC CUTOFFPL component. At Phase $\mathrm{C}$ beside the CUTOFFPL component a soft power law was needed to fit the continuum. A power law with high energy cutoff, the POWEXP model described before, was able to fit the continua of Phases D, E and F. With regard to Phases G, $\mathrm{H}$, I and J, although POWEXP gave a good representation of the continuum, residuals remained at low energy $(E<20 \mathrm{keV})$. We tried to model these residuals by adding another component in the form of a black body or a Comptonization spectrum but we were unable to obtain acceptable fits at all pulse phases. On the other hand we successfully fitted the residuals below $20 \mathrm{keV}$ by using a wide Gaussian component.

Power laws of spectral index $\sim 1$ with high energy cutoff are generally attributed to Comptonization of soft photons in the hot electrons gas of the accretion column (Sunyaev \& Titarchuk 1980). Power law with exponential cutoff have been detected at all phases in the spectrum of Vela X-1, thus suggesting the Comptonization process as the main process responsible for $\mathrm{X}$ ray production.

The nature of the low energy Gaussian bump is however unclear and may suggest that power laws with high energy cutoff as result of Comptonization processes are an oversimplification of the actual complexity of physical processes at the hot spot and that some correction to this simple form have to be expected. Although one could observe that this broad Gaussian correction resembles the Compton reflection of the emitted X-rays by a slab of cold material used to model spectra from AGNs and black hole candidates (Magdziarz \& Zdziaski 1995), attempts to use the reflection spectral models available in XSPEC were not successful. Moreover peaks of the reflected component of the accretion discs should appear above $20 \mathrm{keV}$, while in this case the peak is centered at $13 \mathrm{keV}$. One of the possible reasons resides in the completely different environment that surrounds Vela X-1 with respect to the geometry used by Magdziarz and Zdziaski: that is a cold slab (an accretion disk) as the reflection medium. In Vela X-1 we do not expect the presence of an accretion disk, and the reflection medium is probably the clumpy atmosphere surrounding the neutron star. Also the presence of an Iron line and edge is in favour of this reflection interpretation. Anyway, we will not interpret the Gaussian bump as a second component, but as a simple and "working" correction to the spectral continuum, leaving a detailed discussion to a future paper.

If we interpret the measured continuum as due to Comptonization processes, $E_{\text {fold }}$ is an indication of the mean 
Table 6. Fits results for the Vela X-1 spectra of the pulse Phases G, H, I and J under the hypothesis of the presence of a low energy cyclotron absorption feature. The model used for Phases $\mathrm{G}$ and $\mathrm{J}$ is: CYCLOTRON $\mathrm{CLow} \mathrm{Energy} \mathrm{(LE)}) \times \mathrm{CYCLOTRON}_{[\mathrm{High} \text { Energy (HE)] }} \times[\mathrm{NPEX}(\alpha$ and $\beta$ can assume both positive and negative values)]. For Phases $\mathrm{H}$ and I the model is: CYCLOTRON $_{(\mathrm{LE})} \times \mathrm{CYCLOTRON}_{(\mathrm{HE})} \times \mathrm{NHIGH} \times[\mathrm{POWER}$ LAW]. A constant factor has been taken into account in order to accord HPGSPC and PDS data. The constant was fixed to 1 for the HPGSPC while it was left free for PDS. The uncertainties are given at $3 \sigma$ of confidence.

\begin{tabular}{|c|c|c|c|c|}
\hline parameter & Phase G & Phase $\mathrm{H}$ & Phase I & Phase J \\
\hline $\mathrm{CYCL}_{(\mathrm{LE})}$ Equiv. Width (keV) & $1.3_{-0.3}^{+0.5}$ & $17 \pm 9$ & $10_{-2}^{+5}$ & $6_{-3}^{+10}$ \\
\hline $\mathrm{CYCL}_{(\mathrm{LE})}$ Energy Line $(\mathrm{keV})$ & $24.6 \pm 0.5$ & $20.9 \pm 0.6$ & $21.9 \pm 0.4$ & $23.0 \pm 0.7$ \\
\hline $\mathrm{CYCL}_{(\mathrm{LE})} \sigma(\mathrm{keV})$ & $1.6 \pm 0.8$ & $10_{-2}^{+3}$ & $7.5 \pm 1.5$ & $7 \pm 4$ \\
\hline $\mathrm{CYCL}_{(\mathrm{HE})}$ Equiv. Width $(\mathrm{keV})$ & $20 \pm 5$ & $24_{-6}^{+8}$ & $25 \pm 6$ & $31.5 \pm 5$ \\
\hline $\mathrm{CYCL}_{(\mathrm{HE})}$ Energy Line $(\mathrm{keV})$ & $50.1 \pm 0.5$ & $53.5 \pm 1.5$ & $57 \pm 1$ & $55.5 \pm 0.5$ \\
\hline $\mathrm{CYCL}_{(\mathrm{HE})} \sigma(\mathrm{keV})$ & $6.5 \pm 1.5$ & $8 \pm 2$ & $8.5 \pm 1.5$ & $8 \pm 1$ \\
\hline NPEX $\alpha$ index & $3 \pm 2$ & & & $0.1 \pm 0.1$ \\
\hline NPEX $\beta$ index & $-0.6_{-0.1}^{+1}$ & & & $3.3 \pm 0.5$ \\
\hline NPEX Norm. 2 & $0.02_{-0.02}^{+0.1}$ & & & $\left(3_{-2}^{+7}\right) \times 10^{-5}$ \\
\hline NPEX Folding en. (keV) & $12_{-2}^{+1}$ & & & $6.55 \pm 0.3$ \\
\hline NPEX Norm. ${ }^{a}$ & $7_{-2}^{+1}$ & & & $0.10 \pm 0.02$ \\
\hline POW. LAW Phot. index & & $1.12 \pm 0.03$ & $0.6 \pm 0.1$ & \\
\hline POW. LAW Norm. ${ }^{a}$ & & $0.30 \pm 0.02$ & $0.11 \pm 0.03$ & \\
\hline NHIGH Cutoff en. (keV) & & $17.3 \pm 0.3$ & $11 \pm 2$ & \\
\hline NHIGH Folding en. (keV) & & $12.0 \pm 0.5$ & $11.5 \pm 0.5$ & \\
\hline NHIGH Width (keV) & & $6.5 \pm 1.5$ & $15_{-3}^{+5}$ & \\
\hline PDS constant & $0.86 \pm 0.01$ & $1.15 \pm 0.15$ & $1.06_{-0.5}^{+0.9}$ & $0.94 \pm 0.06$ \\
\hline reduced $\chi^{2}$ & 1.276 & 1.328 & 1.806 & 1.124 \\
\hline d.o.f. & 153 & 153 & 153 & 153 \\
\hline
\end{tabular}

${ }^{a}$ Photons $/ \mathrm{keV} / \mathrm{cm}^{2} / \mathrm{s}$ at $1 \mathrm{keV}$.
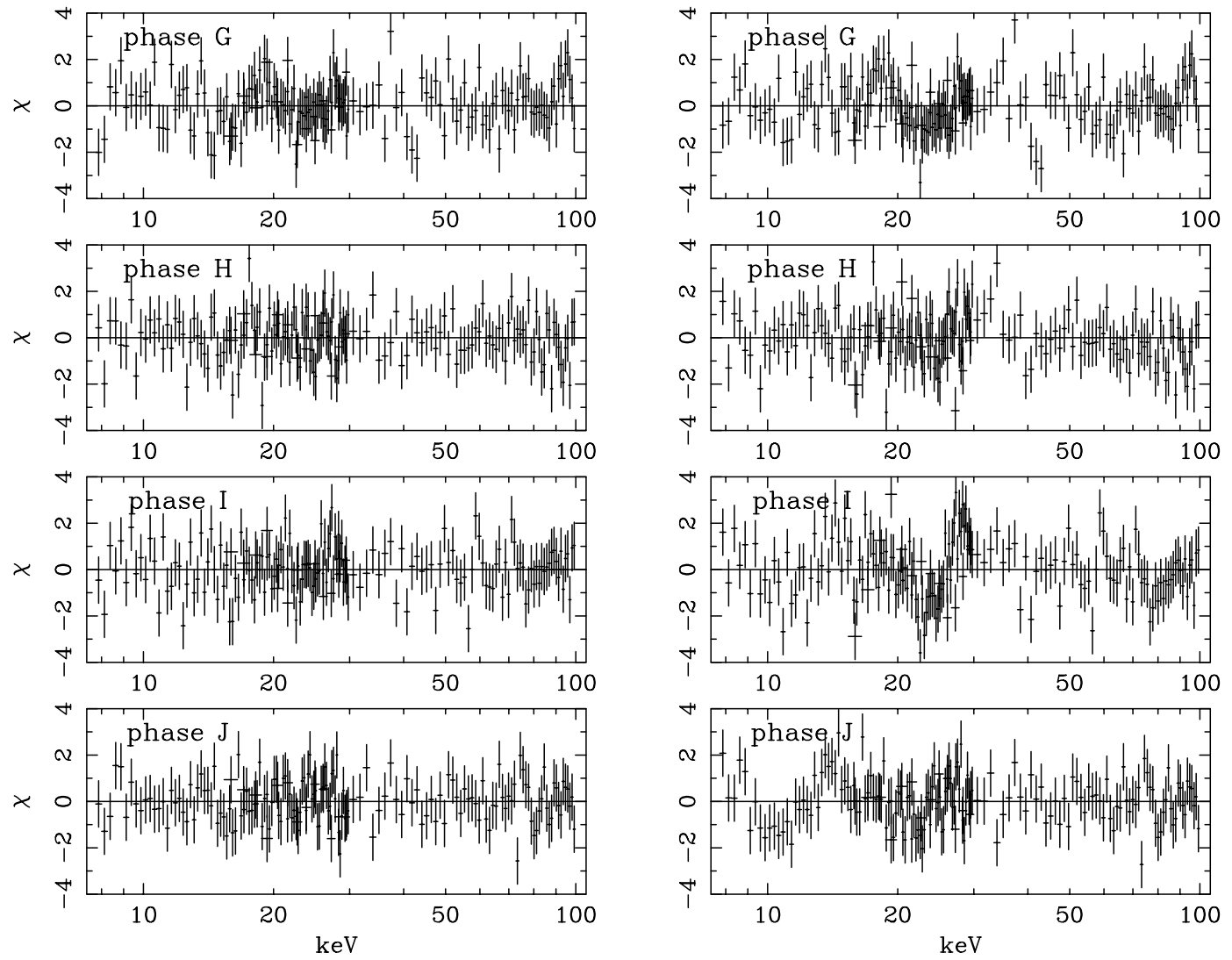

Fig. 11. Comparison between the residuals obtained with the model containing the Gaussian bump (left panels) and the residuals obtained by considering a low energy ( $25 \mathrm{keV})$ cyclotron absorption feature (right panels). 


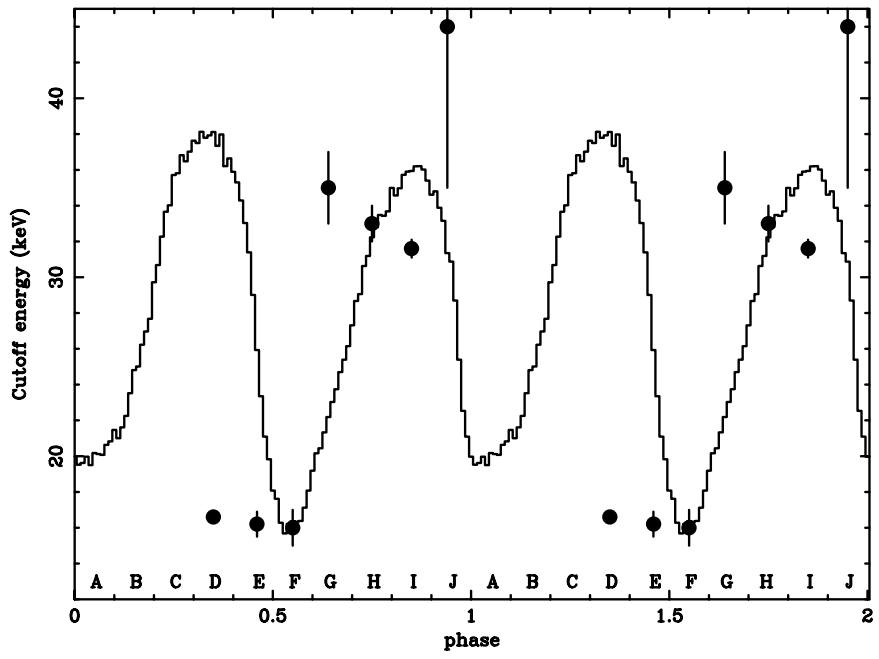

Fig. 12. Values of the cutoff energy as a function of the pulse phase superimposed to the PDS pulse profile (arbitrary units).

temperature of the electrons in the accreting column. Excluding Phases A and B for which the CUTOFFPL model was used, the $E_{\text {fold }}$ appears to be quite uniform in all phases around $\sim 10 \mathrm{keV}$.

The phase dependence of $E_{\text {cut }}$ is shown in Fig. 12. A strong correlation between pulse phase and $E_{\text {cut }}$ is observed. At the first peak (phases 0.3-0.6) $E_{\text {cut }}$ is $\sim 15 \mathrm{keV}$ while at the second peak (phases $0.6-1$ ) $E_{\text {cut }}$ is $\sim 35 \mathrm{keV}$ exceeding $40 \mathrm{keV}$ at descending edge of the peak. In this sense we could refer to a soft and to a hard peak. Furthermore the ratio $E_{\text {cut }} / E_{\text {fold }} \sim 1$ at the first peak suggests an unsaturated Comptonization process (see e.g. Rybicki \& Lightman 1979), whereas the ratio $E_{\text {cut }} / E_{\text {fold }} \sim 3$ at the second peak seems to indicate a saturated Comptonization.

We found high energy residuals at $\sim 55 \mathrm{keV}$ in the spectra of all phase intervals and we have fitted them with Gaussian functions in absorption determining the energy of the centroid, the width of the Gaussian and the equivalent width. We interpreted these features as cyclotron absorption resonance due to the scattering of photons with the Landau levels of the electrons entrapped by the magnetic field lines at the hot spot region. The magnetic field strength is obtained by the cyclotron energy through the relation:

$B_{12}=\frac{1}{\sqrt{1-\frac{2 G M_{\mathrm{NS}}}{R c^{2}}}} \frac{E_{\mathrm{cycl}}}{11.6}$

where $B_{12}$ is the magnetic field in $10^{12}$ Gauss units, $E_{\text {cycl }}$ is the observed cyclotron centroid energy in $\mathrm{keV}, G$ is the gravitational constant, $M_{\mathrm{NS}}$ is the neutron star mass, $R$ its radius and $c$ is the velocity of the light. In the previous formula we have taken into account the gravitational redshift effect. Adopting the neutron star mass of $1.7 M_{\odot}$ as valued by Barziv et al. (2001), we derive magnetic field values ranging from $\sim 6 \times 10^{12}$ to $\sim 7 \times 10^{12}$ Gauss.

In Fig. 13 we plot the behavior of $E_{\text {cycl }}$ as a function of the phase intervals and compare it with the pulsed light curve trend. We observe a strong correlation between $E_{\text {cycl }}$ and the intensity of the pulsation. Generally $E_{\text {cycl }}$ follows the pulsation

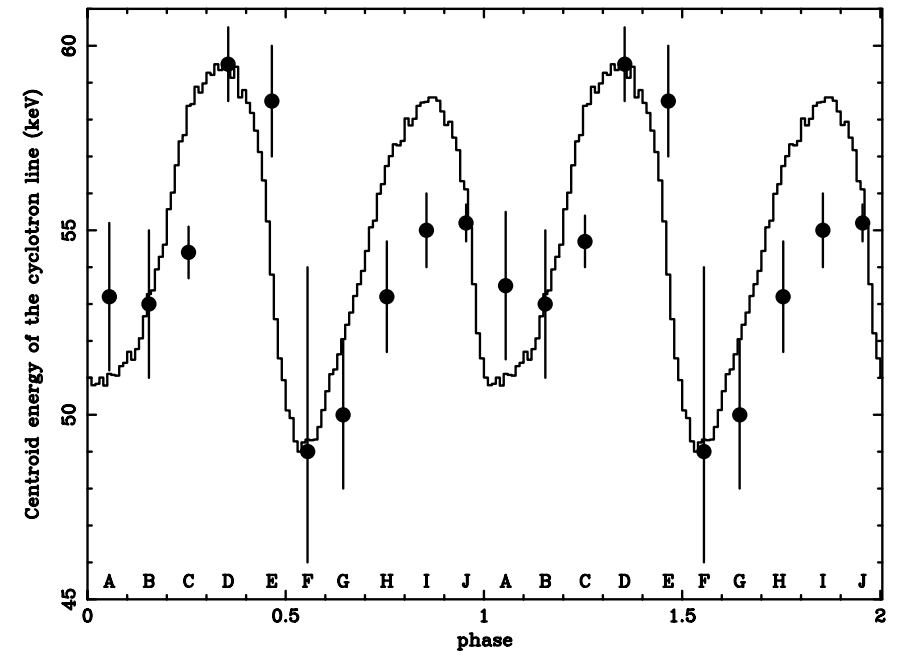

Fig. 13. The centroid energy of the high energy $(\sim 55 \mathrm{keV})$ of the cyclotron absorption feature as a function of the pulse phase superimposed to the PDS pulse profile (arbitrary units).

increasing and decreasing with the luminosity excluding a phase shift between the maximum of luminosity and that one of the magnetic field.

We found variations of the cyclotron line energy up to $10 \mathrm{keV}$. These variations could be attributed to the dependence of the magnetic field on the angle between the magnetic field and the line of sight. In order to explain the observed variations, a polar cap with an angular radius $\sim 45^{\circ}$ degrees wide should be required. This result is in contrast with the typical inferred dimensions of the polar cap. According to theoretical models (see e.g. Davidson \& Ostriker 1973), the area of the neutron star covered by the accreting matter is $\sim 1 \%$ of the whole surface. For spherical accretion the radius of the accretion column is $\sim 10^{5} \mathrm{~cm}$ which corresponds to a few degrees angular radius.

Assuming a vertical structure of the accreting column solves the inconsistence and explains in a very natural way the behavior of the centroid energy of the cyclotron feature. In fact if we assume that the accretion column has a vertical structure as suggested by several authors (see e.g. Wu et al. 1999 and references therein) we could attribute the pulsed light curve and then the phase dependent luminosity to the darkening limb effect due to the different geometrical depths seen. According to this mechanism different phases correspond to different heights of the accreting column and then to different values of magnetic field strength. In particular at the minima of luminosity the accretion column is seen from a lateral point of view, whereas at the maxima of luminosity the accretion column is seen when its geometrical axis forms the smallest angle with the line of sight. Supposing that the strength of the magnetic field decreases with the height, as it is natural for a dipole field structure, we should see stronger magnetic field and then higher cyclotron energy when we have the maximum of luminosity, whereas we should see weaker magnetic field at the luminosity minima.

If this model is correct, we could infer vertical dimension of the accreting column. In fact, supposing a dipolar structure 
for the magnetic field, ignoring relativistic gravitational effects, we could write the following relation:

$\frac{\Delta B}{B}=\frac{\Delta E_{\mathrm{cycl}}}{E_{\mathrm{cycl}}}=3 \frac{\Delta R}{R}$.

In our case, we obtain $\Delta E_{\text {cycl }} \sim 10 \mathrm{keV}$ and a mean value of $E_{\text {cycl }} \sim 55 \mathrm{keV}$. Considering a star radius of $10^{6} \mathrm{~cm}$, we obtain a vertical characteristic length of $\sim 6 \times 10^{4} \mathrm{~cm}$ in good agreement e.g. with the results of Wu et al. (1999) who calculated an accretion column height of $\sim 4.20 \times 10^{4} \mathrm{~cm}$.

Nevertheless this model forecasts a symmetrical behavior with no phase shift of the centroid energy of the cyclotron feature with the pulsation. The observed asymmetry needs other explication. Generally, in order to explain this kind of asymmetry, an offset of the magnetic dipole with respect both to the geometrical centre of the neutron star and to the spinning axis is invoked (see e.g. Leahy 1991; Burderi et al. 2000). The result of the offset is that the region where the magnetic field is stronger does not coincide with the centre of the accreting column so that we can see stronger magnetic field at a different phase with respect to the luminosity maximum.

Another point to be remarked is that, the observed asymmetries of the cyclotron line energy with respect to the pulse profile both in Vela X-1 and in other sources (Santangelo et al. 1999, and references therein) have the characteristic that the maximum of cyclotron centroid energy ever follows the luminosity maximum except Cen $\mathrm{X}-3$ which has an inverted behavior (Burderi et al. 2000). According to the model of the offset there is no reason for privileging a positive or negative phase shift: since the offset should be randomly distributed, we should expect maxima of magnetic field following and anticipating luminosity maxima. A more significative sample of sources is necessary in order to clear if this effect is real or not.

No clear correlation was found between the width of the cyclotron resonance feature and the pulsation as well as between the depth of the line and the pulsation. With regard to the width we found values ranging from $\sim 3$ up to $\sim 10 \mathrm{keV}$. These values could be explained invoking thermal broadening. In this case, assuming a mean temperature $E_{\text {fold }}$ of $10 \mathrm{keV}$ (obtained by the cutoff energy) and a cyclotron energy $E_{\text {cycl }}$ of $55 \mathrm{keV}$, we expect a mean broadening:

$$
\Delta E_{\text {cycl }}=E_{\text {cycl }} \sqrt{E_{\text {fold }} / 511 \mathrm{keV}} \simeq 8 \mathrm{keV} .
$$

The same broadening could be attributed to the distribution of the values of the magnetic field strength in the emission region. In this case the expected broadening is of the order of the $\sim \Delta E_{\text {cycl }}$. Considering the range of values spanned by the cyclotron energy which we measured, we obtain $\Delta E_{\text {cycl }} \sim 10 \mathrm{keV}$. Doppler broadening, due to the infalling velocity of the accretion material, could also be present. In fact near the NS surface the infalling velocity could be a significative fraction of the light speed c ( $v$ of the order of $0.1 \mathrm{c}$ ) (see e.g. Wu et al. $1999)$. Adopting this value, $\Delta E_{\text {cycl }} \sim 0.1 E_{\text {cycl }} \sim 2.5 \mathrm{keV}$ in agreement within a factor 2 or 3 with the values obtained by the fit.

Following the literature we forced our fit to take into account a low energy cyclotron line at about 20-25 keV in those phases for which the bump correction has been needed. Even if the $\chi^{2}$ reduces when we introduce this absorption feature at low energies in substitution of the Gaussian bump, generally we obtained $\chi^{2}$ values worse than those obtained considering the Gaussian bump correction. So we could conclude that the presence of a low energy cyclotron line which some authors would interpret as the fundamental harmonic is mathematically unnecessary to the fit and its appearance could be due to a bad modeling of the continuum shape. However to model the spectral continuum plus lines could be made more difficult by the shape of the fundamental line that we know is not a Gaussian and can be distorted by many effects (Araya \& Harding 1999).

Should this line be present, some strange behavior would characterize this feature. It would appear at energies or below the cutoff energy or very close to that whereas other sources always have cyclotron absorption for energies larger than the cutoff. No clear correlation is observed with the pulsation since the feature involves only the second peak and is absent at the first as well as there is no clear correlation with the high energy cyclotron line. In particular, considering the phases in which the low energy cyclotron line should appear, whereas the high energy cyclotron line follows the pulsation the low energy cyclotron line has an anti-correlated behaviour, roughly decreasing during the increasing of the luminosity and viceversa increasing during the decreasing of the luminosity. Moreover the ratio between the energy of the high energy line and that of the low energy line is always larger than 2 contrary to that observed in other sources in which the ratio between the first harmonic and the fundamental always is less than 2. Furthermore, there is no evidence of a "third" cyclotron harmonic at $\sim 75 \mathrm{keV}$, as we would expect if the feature at $\sim 25 \mathrm{keV}$ were the fundamental cyclotron feature.

\section{Conclusions}

Summarizing, we studied the $8-100 \mathrm{keV}$ pulse phase resolved spectrum of Vela X-1 observed by BeppoSAX. We confirm the complex spectral nature of the continuum for which we used different models for different pulse phase intervals. Comptonization processes could well explain the high energy cutoff which is highly correlated with the pulsation. A cyclotron resonance absorption feature has been observed in all phase intervals, the cyclotron energy varying between 50 and $60 \mathrm{keV}$ corresponding to magnetic fields ranging from $\sim 6 \times 10^{12}$ to $\sim 7 \times 10^{12}$ Gauss. A strong correlation has also observed between the cyclotron energy and the pulsation. With regard to the controversial low energy cyclotron line, we do not find strong evidence of it in our observations. Further observations are required to discern this question.

Acknowledgements. This research has been funded in part by the Italian Space Agency.

\section{References}

Anzer, U., Börner, G., \& Monaghan, J. J. 1987, A\&A, 176, 235

Araya, R. A., \& Harding, A. K. 1999, ApJ, 517, 334

Barziv, O., Kaper, L., van Kerkwijk, M. H., Telting, J. H., \& van Paradijs, J. 2001, A\&A, 377, 925

Boella, G., Butler, R. C., Perola, G. C., et al. 1997a, A\&AS, 122, 299 
Boella, G., Chiappetti, L., Conti, G., et al. 1997b, A\&AS, 122, 327

Börner, G., Hayakawa, S., Nagase, F., \& Anzer, U. 1987, A\&A, 182, 63

Brucato, R. J., \& Kristian, J. 1972, ApJ, 173, L105

Burderi, L., Di Salvo, T., Robba, N. R., La Barbera, A., \& Guainazzi, M. 2000, ApJ, 530, 429

Choi, C. S., Dotani, T., Day, C. S. R., \& Nagase, F. 1996, ApJ, 471, 447

Davidson, K., \& Ostriker, J. P. 1973, ApJ, 179, 585

Deeter, J. E., Boynton, P. E., Lamb, F. K., \& Zylistra, G. 1989, ApJ, 336,376

Dupree, A. K., Gursky, H., Black, J. H., et al. 1980, ApJ, 238, 969

Frontera, F., Costa, E., dal Fiume, et al. 1997, A\&AS, 122, 357

Haberl, F., \& White, N. 1990, ApJ, 361, 225

Jager, R., Mels, W. A., Brinkman, A. C., et al. 1997, A\&AS, 125, 557

Kendziorra, E., Mony, B., Kretschmar, P., et al. 1992, in NASA. Goddard Space Flight Center, The Compton Observatory Science Workshop, 217

Kretschmar, P., Pan, H. C., Kendziorra, E., et al. 1996, A\&AS, 120, 175

Kretschmar, P., Pan, H. C., Kendziorra, E., et al. 1997, A\&A, 325, 623

Kreykenbohm, I., Kretschmar, P., Wilms, et al. 1999, A\&A, 341, 141

Kreykenbohm, I., et al. 2002, private communication

Leahy, D. A., Elsner, R. F., \& Weisskopf, M. C. 1983, ApJ, 272, L256

Leahy, D. A. 1991, MNRAS, 251, 203

Livio, M., Soker, N., de Kool, M., \& Savonije, G. J. 1986, MNRAS, 218, 593

Magdziarz, P., \& Zdziaski, A. A. 1995, MNRAS, 273, 837

Makishima, K., Mihara, T., Nagase, F., \& Murakami, T. 1992, in Frontiers of X-ray Astronomy, ed. Y. Tanaka, \& K. Koyama (Tokyo: Universal Academy Press), 23
Makishima, K., Mihara, T., Nagase, F., \& Tanaka, Y. 1999, ApJ, 525, 978

Manzo, G., Giarrusso, S., Santangelo, A., et al. 1997, A\&AS, 122, 341

Meszaros, P., Nagel W., 1985a, APJ, 298, 147

Meszaros, P., Nagel, W. 1985b, APJ, 299, 138

Mihara, T. 1995, Ph.D. Thesis, University of Tokyo

Nagase, F., Hayakawa, S., Sato, N. 1986, PASJ, 38, 547

Nagase, F. 1989, PASJ, 41, 1

Nagel, W. 1981, ApJ, 251, 278

Orlandini, M. 1993, MNRAS, 264, 181

Orlandini, M., dal Fiume, D., Frontera, et al. 1998, A\&A, 332, 121

Parmar, A. N., Martin, D. D. E., Bavdaz, M., et al. 1997, A\&AS, 122, 309

Rybicki, G. B., \& Lightman, A. P. 1979, Radiative Processes in Astrophysics (New York: John Wiley \& Sons, Inc.)

Sadakane, K., Hirata, R., Jugako, J., et al. 1985, ApJ, 288, 284

Sako, M., Liedahl, D. A., Kahn, S. M., \& Paerels, F. 1999, ApJ, 525, 921

Santangelo, A., Segreto, A., Giarrusso, S., et al. 1999, ApJ, 523, L85

Sato, M., Hayakawa, S., Nagase, F., et al. 1986, PASJ, 38, 547

Soker, N., Livio, M., de Kool, M., \& Savonije, G. J. 1986, MNRAS, 221, 445

Sunyaev, R. A., \& Titarchuck, L. G. 1980, A\&A, 86, 121

van Paradijs, J. A., Hammerschlag-Hensberge, G., van den Heuvel, E. P. J., et al. 1976, Nature, 259, 547

van Paradijs, J. A., Takens, R., \& Zuiderwijk, E. 1977, A\&AS, 30, 195

White, N. E., Swank, J. H., \& Holt, S. S. 1983, ApJ, 270, 711

Wu, H., Lin, X., Xu, H., \& You, J. 1999, ChA\&A, 23, 498 